\title{
Smart Door / COVID-19 Face Mask Detection
}

\author{
Pavan Narayana A, Janardhan Guptha S, Deepak S, Pujith Sai P
}

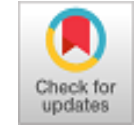

Abstract: January 27 2020, a day that will be remembered by the Indian people for a few decades, where a deadly virus peeped into a life of a young lady and till now it has been so threatening as it took up the life of 3.26 lakh people just in India. With the start of the virus government has made mandatory to wear masks when we go out in to crowded or public areas such as markets, malls, private gatherings and etc. So, it will be difficult for a person in the entrance to check whether everyone one are entering with a mask, in this paper we have designed a smart door face mask detection to check whether who are wearing or not wearing mask. By using different technologies such as Open CV, MTCNN, CNN, IFTTT, ThingSpeak we have designed this face mask detection. We use python to program the code. MTCNN using Viola- Jones algorithm detects the human faces present in the screen The and then finds the location on the colored image. In this algorithm MTCNN first detects the face in grayscale image locates it and then finds this location on colored image. CNN for detecting masks in the human face is constructed using sample datasets and MobileNetV2 which acts as an object detector in our case the object is mask. ThingSpeak is an open-source Internet of things application used to display the information we get form the smart door. This deployed application can also detect when people are moving. So, with this face mask detection, as a part to stop the spread of the virus, we ensure that with this smart door we can prevent the virus from spreading and can regain our happy life.

Keywords: CNN, IFTTT, MTCNN, Open CV, ThingSpeak.

\section{INTRODUCTION}

A few nations around the globe have utilized face masks obligatory out in the open to help control the spread of COVID-19, the sickness brought about by the novel coronavirus. A month ago, the WHO refreshed its direction on masks, suggesting that they be worn in open regions where there is a danger of boundless network transmission and

Manuscript received on July 19, 2021.

Revised Manuscript received on July 25, 2021.

Manuscript published on July 30, 2021.

* Correspondence Author

Pavan Narayana A, Studying BTech (3rd year), Department of Electronics and Communication Engineering (Specialization in IoT \& Sensors), Vellore Institute of Technology, Vellore (Tamil Nadu), India. Email: pavan.narayana2018@vitstudent.ac.in

Janardhan Guptha $\mathbf{S}^{*}$, Studying BTech (3rd year), Department of Electronics and Communication Engineering (Specialization in IoT \& Sensors), Vellore Institute of Technology, Vellore (Tamil Nadu), India. Email: janardhan.guptha2018@vitstudent.ac.in

Deepak S, Studying BTech (3rd year), Department of Electronics and Communication Engineering (Specialization in IoT \& Sensors), Vellore Institute of Technology, Vellore (Tamil Nadu), India. Email: sdeepak.2018@vitstudent.ac.in

Pujith Sai P, Studying BTech (3rd year), Department of Electronics and Communication Engineering (Specialization in IoT \& Sensors), Vellore pujithsai.p2018@vitstudent.ac.in

(c) The Authors. Published by Blue Eyes Intelligence Engineering and Sciences Publication (BEIESP). This is an open access article under the CC BY-NC-ND license (http://creativecommons.org/licenses/by-nc-nd/4.0/) Viola-Jones algorithm first detects the face on the grayscale image Institute of Technology, Vellore (Tamil Nadu), India. Email:

Physical separating is troublesome, for example, on open vehicle, in shops or other shut settings.so we thought bringing a smart door into the market which detects if a person wearing a mask or not is crucial for the society.

One day we should come back to our normal lives. To do that we need to stop the spread of virus, to make that possible everyone should wear mask. So we are implementing this face mask detection using CNN and MTCNN algorithms. We use OpenCV - OpenCV is a library of programming functions mainly aimed at real-time computer vision, used to acquire images from web cam or other camera modules. MTCNN - Multi-task Cascade Convolutional Neural Network, used to detect a human face in an image and also used to localize the face in that image

CNN-Convolutional Neural Network, used to detect if a person is wearing Mask or not. IFTTT, If This Then That, also known as IFTTT, is a freeware web-based service that creates chains of simple conditional statements, called applets - used to send notification to smart phone when a person is detected, uses HTTP POST request to send the required message. ThingSpeak is an open-source Internet of Things application and API to store and retrieve data from things using the HTTP and MQTT protocol over the Internet or via a Local Area Network - Used to collect data about no. of people wearing or not wearing masks. We use a Teachable machine to train the mask detector model.

So with this we can deploy the face mask detection and can detect people who or wearing masks and who are not wearing and can prevent spread of the virus.

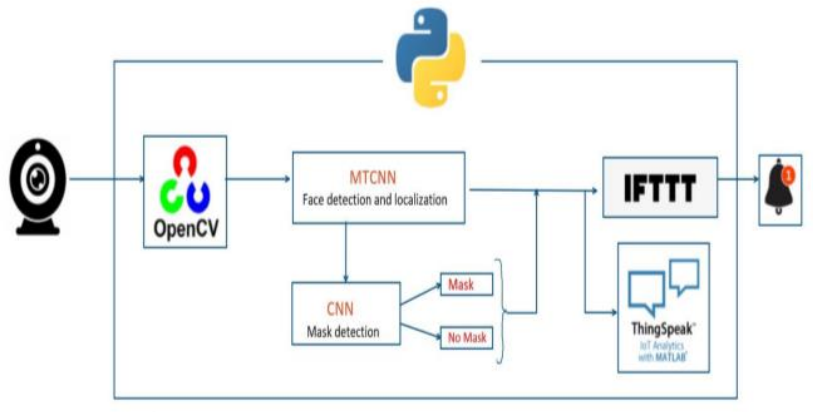

Figure 1: Brief methodology of Face mask detection system.

From Figure 1 we can see the brief methodology how the proposed system works. We acquire images by using Open CV connected to the camera, then MTCNN detects the face, CNN detects whether person is wearing mask or not. By using IFTTT we get the notification and Thing Speak is used to display the information

\section{LITERATURE REVIEW}

[1] This paper explains about the Convolutional Neural networks.

Published By:

Blue Eyes Intelligence Engineering and Sciences Publication

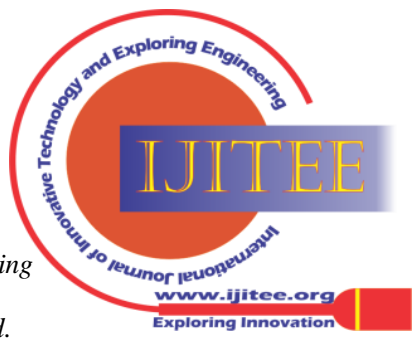


At present CNN plays a major role in machine learning for many applications like image and video recognitions. This paper discusses about the Convolution, Stride, Padding, Input representations, Features of CNNs, Convolutional Formula and Pooling.

[2] This paper explains about the Cascaded Convolution neural networks for joint face detection. This Cascaded CNNs helps in improving the performance in detection and alignment. This paper discusses about the CNN Training using Face Classification, facial landmark localization and Bounding Box Regression.

[3] This paper discusses about Social distance Monitoring and Face mask detection based on Convolution Neural Network (CNN) based binary classifier and object detection. YOLOv3, DBSCAN and DSFD are the Deep learning models used in this paper.

[4] This paper proposed a Face mask detection system using the three main concepts, they are Deep learning with FPN to detect the Human, Multi-Task Convolutional Neural Networks (MT-CNN) to detect human faces and convolutional neural network classifier to recognize masked and unmasked people.

[5] In this paper using CNN and mobile Net V2 architecture they have deployed the face mask detectors. Python programming language is used for implementation of the deployed model and they used datasets of tensor flow and keras. By using those datasets collected they trained their face mask model using python script.

[6] To help the current COVID situation they have developed the face mask detection which is deployed in a door, which finally will be a smart door. This paper is developed using artificial intelligence model installed with a camera and attached to a raspberry pi device. Using the mobile, they displayed the information required. They used about 1376 pictures containing with and without masks by using the CNN model.

[7] This paper deploys the face mask model for the present health care system. Using technologies like Tensor flow and open cv they have deployed the face mask detection model where a square box is drawn with different colour for the people who are wearing mask and who are not wearing. Image processing concepts are also used in the deployed model.

[8] Considering the public safety and health precautions in this paper they have used the simplest ways for deploying the face mask detection model. The model that has been proposed in this paper is trained using Kaggle data sets. Scikit learn and CNN are used to implement the model.

[9] In this paper, to extract deep features from images of faces they have worked and studied on different deep Convolution Neural Networks. Some of these deep features have been further developed and processed using different machine learning classifiers like K-Nearest Neighbors (K-NN) and Support Vector Machine (SVM). They have worked using different models as mentioned before and calculated the efficiency and accuracy of each and every model and they have noticed that the best classification rate is $97.1 \%$ this was achieved when implementing by combining MobileNetV2 model and SVM.

[10] In this paper, the authors have developed a face detection model which when detects that a person is not wearing mask rings an alarm that someone is not wearing mask and alerts the people in that region. In this model the human face is detected by Haar cascade classifier and the whether the face has a mask or not is detected by YOLOv3 algorithm. The complete model along with the hardware requirements have been completed and implemented in an office environment at the entrance and this model is working with an accuracy of $90.1 \%$. This accuracy in real-time environment is very good, hence we can conclude that is also a good reference model that can be taken into consideration before developing mask detection models.

[11] In this paper using data augmentation and model compressing and transfer learning methods they have proposed a detection model PP-YOLO-mask based on PP-YOLO. The single picture recognition speed of the mask detection model is $11.842 \mathrm{~ms}$ and public scene mask detection accuracy is nearly $86.69 \%$ these shows that their system is good and accurate. This model when compared with Faster RCNN and YOLOv3 the experimental results conclude that the detection speed and accuracy compared to the above-mentioned models is quite high and thus system is hence more effective compared to the other models.

[12] In this paper they have tried the three algorithms: KNN, Mobile Net and SVM and they have tried implementing the mask detection model using all the three algorithms and tried comparing the performance of the three mask detection models developed using all the three algorithms and compare their recognition time and accuracy and they tried to conclude which model is working more efficiently and accurately in real-time applications. While trying these different models developed by different algorithms, they have found that for the given input video or images the model implemented using the Mobile Net algorithm is more accurate and precise and hence they concluded that it is the best algorithm for real-time application of this mask detection model.

[13] In this paper they have taken around 848 sample images with a resolution of $416 \times 416$ pixels and constructed a Convolution Neural Network (CNN). Based on the performance of the trained model that they have implemented they have tested it in real-time environment in front of a campus building during an open-house event and calculating the metrics they have found that this model is accurate up to $81 \%$. Hence this is also one of the good mask detection models with god accuracy developed by the authors and since they have also implemented in real-time environment $81 \%$ accuracy is very good.

[14] In this paper they have implemented a mask detection model which processes videos using deep learning and tells whether someone is wearing mask or not. This model mainly depends on the MTCNN face detection model which detects human faces in an image and stores their corresponding four landmarks around the face.

Images in which the human faces have been detected are later processed by neoteric classifier and MobileNetV2 which acts as an object detector in this case the object is mask and identifies these regions in which the mask is present and concludes which face is wearing mask and which faces are not wearing.

This method has been caught emitting good accuracy and precision while tested against a video sample which consists of video samples or images from public places complying with COVID-19 protocols.

Blue Eyes Intelligence Engineering and Sciences Publication 
Hence this method of mask detection is effective and can be used for real-time detection.

[15] In this paper they have introduced mask detection technique done using Matlab. Data set allocation and R-CNN algorithms work more efficiently according to author when integrated with Matlab. Complex pictures with large crowds and low light are dealt in this model of mask detection using Matlab. The Faster R-CNN algorithm is generally used in complex security and medical systems. Lot of complexities like colour changes, contrast changes, brightness changes, balanced face restricting are dealt in this model using Matlab. The researchers who are involved in this work have mainly concentrated on the complexities in these face detection models generally and taken it as a challenge to improve the models efficiency instead of these challenges, hence this a good and unique attempt to take up the difficulties and sort out the challenges for future use and development of these mask detection models and they have chosen Matlab as the new inclusion in order to achieve this feat

\section{PROPOSED WORK}

In this project we use PYTHON code to detect number of people standing outside and whether they are wearing masks or not. In general, in real-time application Open CV captures the real-time images for processing but in our case, we will be wearing or not wearing masks.

As we have mentioned in technology MTCNN is used to project so we import the MTCNN library for this in our application. We decide whether the particular person is wearing a mask or not using CNN. As we include the mask model trained by us using this CNN checks whether the face detected by MTCNN has a mask or not.

If there is no mask it draws a red box around the face and if there is a mask it draws a green mask. The logic behind drawing these green and red boxes exactly around the face is when MTCNN detects a face it considers it as a 2-D object on geometrical plane and it assumes four points around the face and considers then as the face borders.

Now we are joining these points and changing the lines RGB values i.e., Red, Blue and green components of its color mixture to $(0,255,0)$ I.e., Red value of color is set to zero, Green 255 which is maximum and Blue is also set to zero which results in a green color box around the face indicating that he or she is wearing mask for visualizing and when mask is not detected in a particular face, we change the RGB value I.e., Red, Green, Blue components to $(255,0,0)$ indicating red value 255 maximum, Green zero and Blue is also set to zero results in a red color box around the face indicating that he or she is not wearing mask.

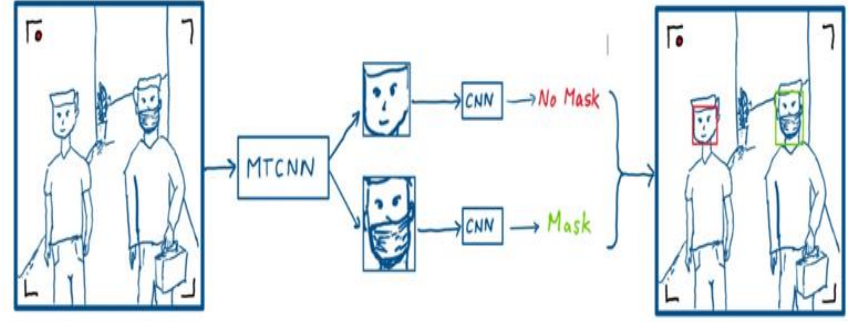
detection. providing a video with some frames with pictures of people detect a human face in a frame the same happens in our

Figure 2. Process of MTCNN and CNN for Mask

Figure 2 describes the MTCNN and CNN process for mask detection where red box denotes person is not wearing mask and green box denotes that person is wearing mask.

Initially in our python application we initialize 2 variables count_mask and count_no_mask as zero. Whenever CNN detects a face with mask, we increase the count_mask value by 1 using logic count_mask = count_mask +1 . When there is no mask, we increase count_no_mask value by using logic count_no_mask = count_no_mask +1 . So, we get the total number of people count by adding count_mask and count_no_mask.

As we know when we create an applet in IFTTT it has an If This Then That logic and we need to provide that condition like "If This happens Then execute That" In this application at If we take a web request using webhooks and whenever we receive a web request, we execute Then That field which sends notifications. The three values I.e., total number of people, count_mask and count_no_mask is sent to IFTTT using the webhooks web request URL as value1, value2, value 3 and these values are used to construct the message in the notification sent to our phone. Example: " 8 " people are standing at the door "4" are wearing mask and "4" are not wearing mask. Value 2 and value 3 I.e., count_mask and count_no_mask is sent to ThingSpeak using the ThingSpeak channels write API key of the channel that we have created for this mask detection. The count_mask value is displayed in the form of graph in Field1 and count_no_mask is displayed in form of graph in Field2 in our ThingSpeak channel

\section{METHODOLOGY}

\section{Training of mask detection CNN:}

We used Teachable Machine to train the Mask Detector model (which is two class classification problem) that can classify if a person is wearing a mask or not wearing a mask. Teachable Machine is a web-based tool from Google that makes creating machine learning models fast, easy, and accessible to everyone. Teachable Machine uses a MobileNetv2 to train models using transfer learning approach to train only the last two DENSE layers and uses a SoftMax activation function for multiple class classification. Mobile Nets are small, low-latency, low-power models parameterized to meet the resource constraints of a variety of use cases.

In Figure 3 we have tested our constructed CNN model for mask detection constructed using the image samples in class 1 and class 2 as we can see in the figure in fig 3 we can see that it shows in the output $99 \%$ for No mask indicating that the provides sample doesn't have mask. In Figure 4 we have drawn the mask on the same face and this time in the output It shows $100 \%$ in the mask section and $0 \%$ in No mask section indicating that this face has a mask. Similarly, have tested taking many other samples and got the accurate results

So, we can conclude from this testing that our CNN model has been constructed successfully for detecting whether someone is wearing mask or not

Published By:

Blue Eyes Intelligence Engineering and Sciences Publication

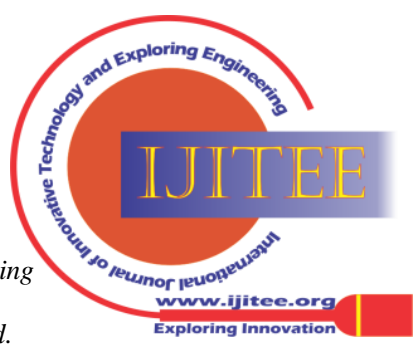




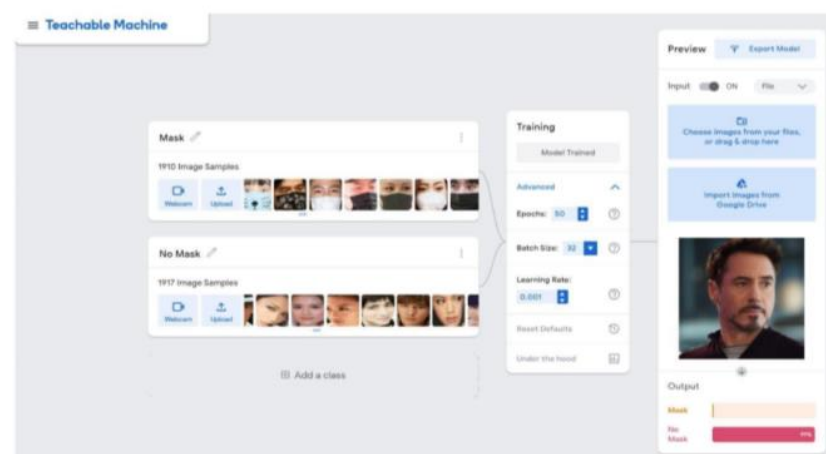

Figure 3: Constructed CNN model for mask detection (unmasked human).

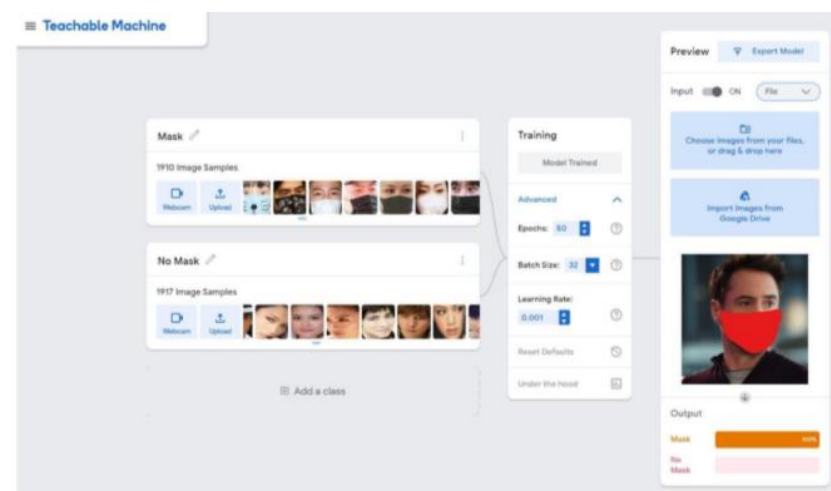

Figure 4: constructed CNN model for mask detection (masked human).

Table 1. Accuracy details in Teachable Machine.

\begin{tabular}{|c|c|c|}
\hline Class & Accuracy & \#samples \\
\hline Mask & $\mathbf{0 . 9 9}$ & $\mathbf{5 0}$ \\
\hline No Mask & $\mathbf{1 . 0 0}$ & $\mathbf{5 0}$ \\
\hline
\end{tabular}

In Table 1 We have given training using 50 photos with masks and 50 without masks while we have tested using the same samples, we got an accuracy of 0.99 for samples with masks and 1.00 for the samples without mask

\section{RESULT}

We aim to successfully stop the spread of virus and that can be achieved by using this face mask detection, where wearing mask can lead to stop the spread of virus. So we have successfully achieved our Smart door face mask detection using MTCNN and CNN technologies.

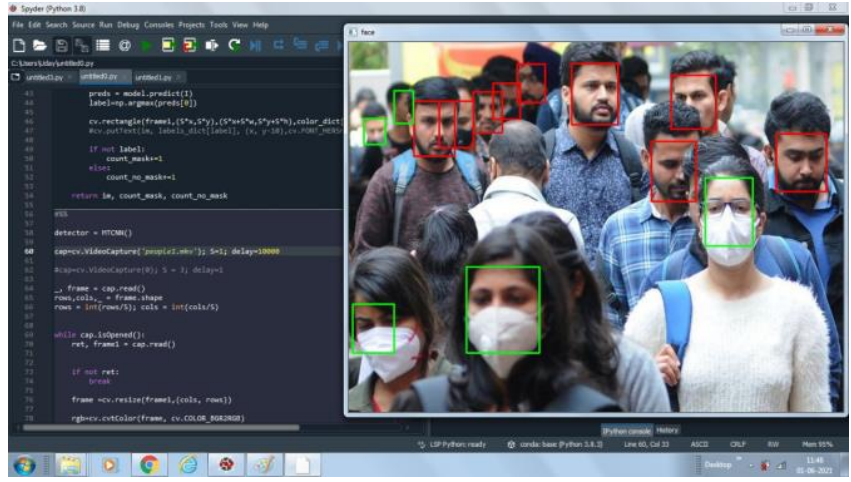

Figure 5 : Software Simulation of the project

In Figure 5 we can see a group of people with and without masks, we can see that the red bounded box around the human face indicates that he is not wearing mask and green box bounded around the human face indicates that he is wearing mask.
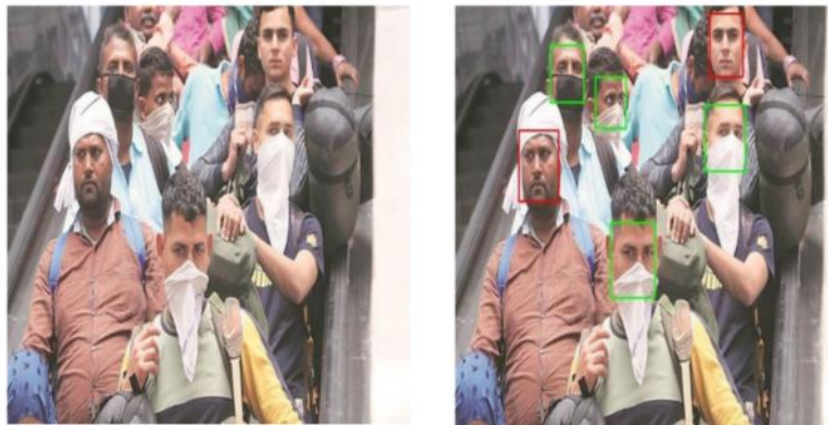

Figure 6 : Mask detection system

In Figure 6 we can see the difference between before detection and after detection of mask.

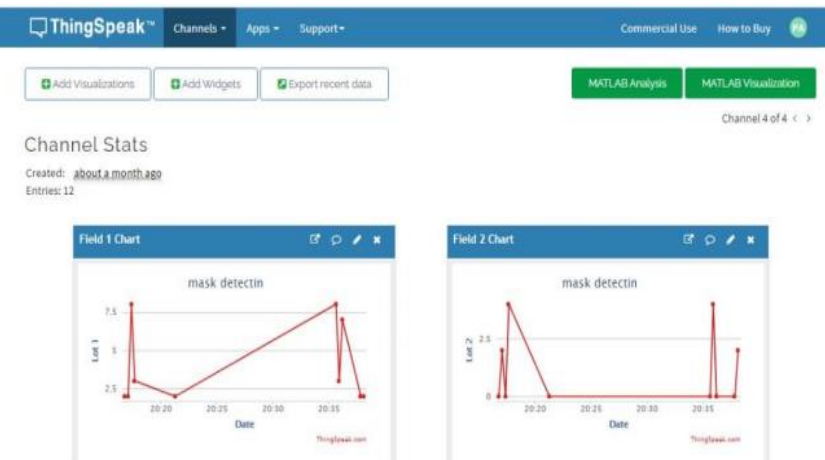

Figure 7 : Thinkspeak channel .

Finally Figure 7 gives us the visualization in the ThingSpeak, how many are wearing mask in the field 1 and how many are not wearing mask in the field 2 .

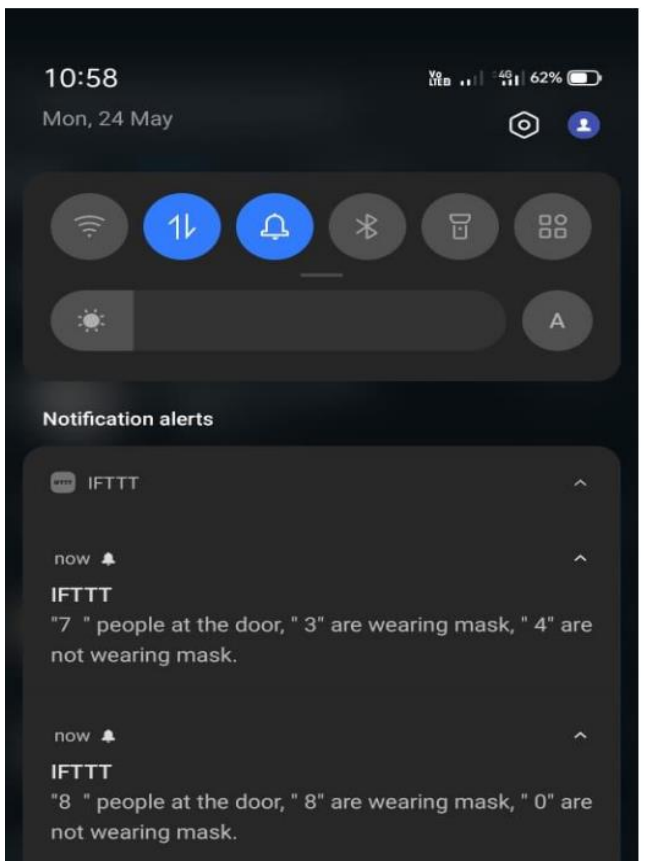

Figure 8: Mobile notifications

Published By:

Blue Eyes Intelligence Engineering and Sciences Publication (C) Copyright: All rights reserved. 
Figure 8 shows us the notification that we get to our phone using the IFTTT applet describing how many people are in front of the door, how many are wearing mask and how many are not wearing mask.

\section{CONCLUSION}

We, as a part of our country and responsibility we have, came up with the idea of smart door/face mask detection and have successfully implemented it to stop the spread of COVID19. We have successfully developed interactive mask detection model using MTCNN, CNN, IFTTT, Things peak which is showing accurate results and the salient and feasible feature that we included is connecting it with IFTTT so that the notifications can be sent to mobile of concerned authority or observer this triggers and alerts the concerned user and helps them in maintaining their environments safe and secure. Our mask detection model when tested against the samples that we have used for training has shown high accuracy around 0.99 for samples with masks and 1.00 for without mask we can ensure that even when we use this model in real-time environment we can get good accuracy. People who are not infected by the virus can be benefited by avoiding the contact from the people who are infected. If most of the people use these smart doors in their shops, homes and in different industries, people who are not wearing masks will automatically recognized and will not be allowed inside. So, the usage of masks will become mandatory which will help preventing the spread of the virus

\section{FUTURE SCOPE}

We can implement this model in such a way that If we add face recognition along with mask detection, we can get the details of the people who are not wearing masks and when it comes to school or working office premises, we this will be very useful. We can record the details of number of particular persons caught without wearing a mask and we can take disciplinary actions on them. This would be very useful and we can store this data and understand metrics like how well the people are following COVID-19 guidelines. Big data with AI and ML models have a lot of scope. We can also use this in immigration centers like airports or Bus stations so that whenever someone is not wearing a mask is detected it we can set an alarm to trigger and this would alert the security guards or people in that region so that they can be safe

\section{REFERENCES}

1. Saad ALBAWI , Tareq Abed MOHAMMED, Saad AL-ZAWI,

2. Kaipeng Zhang, Zhanpeng Zhang, Zhifeng Li, "Joint Face Detection and Alignment Using Multitask Cascaded Convolutional Networks", IEEE SIGNAL PROCESSING LETTERS, VOL. 23, NO. 10, OCTOBER 2016.

3. Sahana Srinivasan, Rujula Singh R, Ruchita R Biradar, Revathi SA, "COVID-19 Monitoring System using Social Distancing and Face Mask Detection on Surveillance video datasets", Proceedings of International Conference on Emerging Smart Computing and Informatics (ESCI), IEEE 2021.

4. Shay E. Snyder, Ghaith Husari, "Thor: A Deep Learning Approach for Face Mask Detection to Prevent the COVID-19 Pandemic", SoutheastCon Conference IEEE 2021.

5. Shena Sakhi, Ajay Kumar,Guptha, Sudeept Singh Yadav, Uttam Kumar, "Face Mask Detection System using CNN", International Conference on Advance Computing and Innovative Technologies in Engineering(ICACITE) ,IEEE 2021

6. Baluprithviraj.K.N, Bharathi.K.R, Chendhuran.S, Lokeshwaran.P, "Artificial Intelligence based Smart Door with Face Mask Detection", "Understanding of a Convolutional Neural Network", ICET,IEEE 2017

Proceedings of the International Conference on Artificial Intelligence and Smart Systems (ICAIS) IEEE 2021.

7. Harish Adusumalli, D. Kalyani, R.Krishna Sri, M.Pratapteja, P V R D Prasada Rao, "Face Mask Detection Using OpenCV",Proceedings of the Third International Conference on Intelligent Communication Technologies and Virtual Mobile Networks (ICICV ), IEEE 2021.

8. Suresh K, Palangappa MB , Bhuvan S, "Face Mask Detection by using Optimistic Convolutional Neural Network", Proceedings of the Sixth International Conference on Inventive Computation Technologies [ICICT], IEEE 2021

9. Abdellah Oumina, Noureddine El Makhfi, Mustapha Hamdi, "Control The COVID-19 Pandemic: Face Mask Detection Using Transfer Learning" , 2nd International Conference on Electronics, Control, Optimization and Computer Science (ICECOCS), IEEE 2020|.

10. Truong Quang Vinh, Nguyen Tran Ngoc Anh, "Real-Time Face Mask Detector Using YOLOv3 Algorithm And Haar Cascade Classifier", International Conference on Advanced Computing And Applications (ACOMP) IEEE 2020.

11. Wang Jian, Lin Lang, "Face mask detection based on Transfer learning and PP-YOLO", 2nd International Conference on Big Data, Artificial Intelligence and Internet of Things Engineering (ICBAIE), IEEE 2021.

12. Wuttichai Vijitkunsawat, Peerasak Chantngarm, "Study of the Performance of Machine Learning Algorithms for Face Mask Detection", IEEE 2020.

13. Ponlawat Khamlae, Kingkarn Sookhanaphibarn, and Worawat Choensawat, "An Application of Deep-Learning Techniques to Face Mask Detection During the COVID-19 Pandemic" , 3rd Global Conference on Life Sciences and Technologies, IEEE 2021

14. Aniruddha Srinivas Joshi, Shreyas Srinivas Joshi, Goutham Kanahasabai , Rudraksh Kapil , Savyasachi Gupta, "Deep Learning Framework to Detect Face Masks from Video Footage", 12th International Conference on Computational Intelligence and Communication Networks , IEEE 2020.

15. S. Meivel, K. Indira Devi, S. Uma Maheswari, J. Vijaya Menaka, "Rea time data analysis of face mask detection and social distance measurement using Matlab", Materials Today: Proceedings, ELSEVIER 2020.

16. X. Zhu and D. Ramanan, "Face detection, pose estimation, and landmark localization in the wild," in IEEE Conf. Comput. Vis. Pattern Recognit., 2012, pp. 2879-2886

17. A. Krizhevsky, I. Sutskever, and G. E. Hinton, "ImageNet classification with deep convolutional neural networks," in Adv. Neural Inf. Process. Syst., 2012, pp. 1097-1105.

18. O. Abdel-hamid, L. Deng, and D. Yu, "Exploring Convolutional Neura Network Structures and Optimization Techniques for Speech Recognition," no. August, pp. 3366-3370, 2013

19. B. J. Cowling, K.-H. Chan, V. J. Fang, C. K. Cheng, R. O. Fung, W. Wai, J. Sin, W. H. Seto, R. Yung, D. W. Chu et al., "Facemasks and hand hygiene to prevent influenza transmission in households: a cluster randomized trial," Annals of internal medicine, vol. 151, no. 7, pp. 437-446, 2009.

20. S. W. Sim, K. S. P. Moey, and N. C. Tan, "The use of facemasks to prevent respiratory infection: a literature review in the context of the health belief model," Singapore medical journal, vol. 55, no. 3, p. 160, 2014

\section{AUTHORS PROFILE}

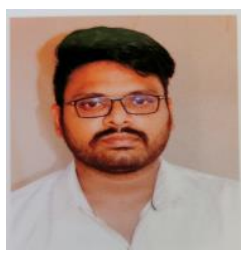

Pavan Narayan A, presently studying BTech, Electronics and Communication Engineering (Specialization in IoT \& Sensors) in Vellore Institute of Technology, Vellore (Tamil Nadu), India. His research mainly includes applications based on Internet of Things, published research papers on Mobile Controlled Robot and Home Automation.

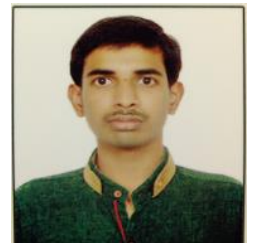

Janardhan Guptha S, presently studying BTech, Electronics and Communication Engineering (Specialization in IoT \& Sensors) in Vellore Institute of Technology, Vellore (Tamil Nadu), India. His research includes Internet of Things \& embedded systems and recently published a paper based on its application.

Published By:

Blue Eyes Intelligence Engineering and Sciences Publication

(C) Copyright: All rights reserved.

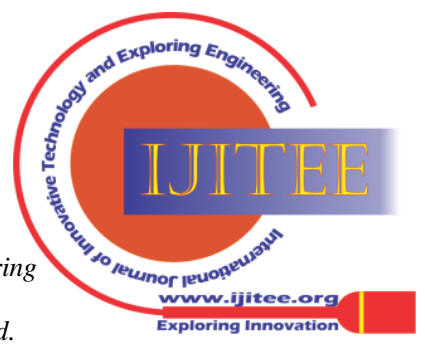



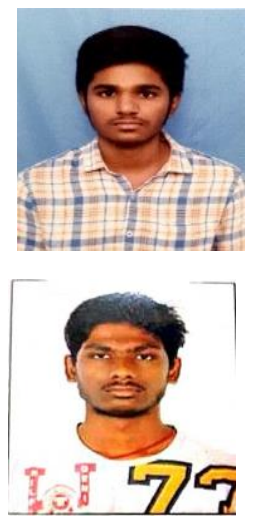

Deepak S, presently studying BTech, Electronics and Communication Engineering (Specialization in IoT \& Sensors) in Vellore Institute of Technology, Vellore (Tamil Nadu), India. His area of interest relates to sensors, microcontrollers and various projects dealing with these fields have been accomplished.

Pujith Sai P, presently studying BTech, Electronics and Communication Engineering (Specialization in IoT \& Sensors) in Vellore Institute of Technology, Vellore (Tamil Nadu), India. His area of research and interest lies in Iot applications, embedded systems and java, python application

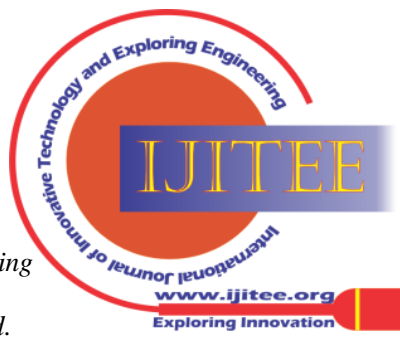

